\title{
Research Article \\ Effect of Ge Nanoparticles in the Core of Photonic Crystal Fiber on Supercontinuum Generation
}

\author{
Seongmin Ju, ${ }^{1}$ Yuseung Lee, ${ }^{1}$ Seongmook Jeong, ${ }^{2}$ Youngwoong Kim, ${ }^{3}$ In-Sik Kim, ${ }^{4}$ \\ Do-Kyeong Ko, ${ }^{1}$ and Won-Taek Han ${ }^{1}{ }^{1}$ \\ ${ }^{1}$ School of Electrical Engineering and Computer Science/Department of Physics and Photon Science, Gwangju Institute of Science \\ and Technology, 123 Chemdangwagi-ro, Buk-gu, Gwangju 61005, Republic of Korea \\ ${ }^{2}$ Laser R\&D Laboratory, LIG Nex1, 207 Mabuk-ro, Giheung-gu, Yongin-si, Gyeonggi-do 16911, Republic of Korea \\ ${ }^{3}$ Nano-Photonics Research Center, Korea Photonics Technology Institute, 9 Chemdanventure-ro, 108 Beon-gil, Buk-gu, \\ Gwangju 61007, Republic of Korea \\ ${ }^{4}$ Molecular Biophysics and Integrated Bioimaging Division, Lawrence Berkeley National Laboratory, Berkeley, CA 94720, USA
}

Correspondence should be addressed to Won-Taek Han; wthan@gist.ac.kr

Received 20 December 2018; Accepted 7 May 2019; Published 16 May 2019

Guest Editor: Matthieu Roussey

Copyright (C) 2019 Seongmin Ju et al. This is an open access article distributed under the Creative Commons Attribution License, which permits unrestricted use, distribution, and reproduction in any medium, provided the original work is properly cited.

The effect of Ge nanoparticle (Ge NP) incorporation in the germanosilicate glass core of the photonic crystal fiber (PCF) on supercontinuum generation (SCG) was investigated. The Ge NP-doped germanosilicate glass core PCF was fabricated by using the modified chemical vapor deposition (MCVD) and the stack-and-draw processes. The average diameter of Ge NPs embedded in the core of the PCF was $4.2 \mathrm{~nm}$. The absorption peaks at $480 \mathrm{~nm}$ and $515 \mathrm{~nm}$ and the band from 600 to $800 \mathrm{~nm}$ were attributed to the Ge NPs in the core of the PCF. SCG of the $490 \mathrm{~nm}$ bandwidth ( $598 \mathrm{~nm} \sim 1088 \mathrm{~nm}$ ) with a conversion efficiency of $31 \%$ was obtained by pumping at $800 \mathrm{~nm}$ with the Ti:sapphire femtosecond laser of $160 \mathrm{~mW}$ with $35 \mathrm{fs}$ pulse at $1 \mathrm{kHz}$ repetition rate, resulting from the enhanced optical nonlinearity from Ge NPs as well as the PCF structure of the fiber.

\section{Introduction}

Supercontinuum generation (SCG) is a spectral broadening phenomenon to yield a broadband output when a narrow-band incident laser pulse goes through an optically nonlinear medium. A broadband supercontinuum laser source generated by injection of high-power ultrashort pulses into a nonlinear medium has much attention in the applications including optical coherence tomography, time-resolved spectroscopy, optical frequency metrology, and optical communications [1-12]. Particularly, SCG based on highly nonlinear optical fiber has been of interest due to its low optical loss and high stability and reliability. Recently, various types of optical fibers were used for the SCG: conventional highly nonlinear fiber, photonic crystal fiber (PCF), tapered fiber, and specialty fibers incorporated with the nanoparticles
(NPs) of semiconductors, heavy metals, and oxides [16, 13-18]. Among these nonlinear optical fibers, the PCFs and the small core fibers have turned out to be interesting because of their very large nonlinearity and controllability of chromatic dispersion. Recently, our group has reported the SCG with a spectral bandwidth of $400 \mathrm{~nm}$ using the specialty optical fiber incorporated with Si NPs in the core [14]. It is well known that group IV semiconductor NPs such as Si and Ge have a large optical nonlinearity due to the formation of nonbridging oxygens (NBOs) and defects and due to the strong quantum confinement effect of the NPs [14, 1823]. Particularly, Ge NPs have stronger quantum confinement and higher optical nonlinearity than Si NPs resulting from its direct-gap semiconductor nature [20, 23-26]. Therefore, in this paper, we fabricated the germanosilicate glass core PCF incorporated with Ge NPs 
and investigated its SCG characteristics upon pumping with the femtosecond laser.

\section{Experiments}

The Ge NP-doped core PCF was fabricated by using the modified chemical vapor deposition (MCVD), which is based on the high temperature oxidation of reagents inside a rotating silica glass tube by heating using an external heat source, and the stack-and-draw methods $[27,28]$. The schematic diagram of the fabrication steps of the Ge NP-doped core PCF is shown in Figure 1. Porous germanosilicate layers were deposited onto the inner surface of a silica glass tube by the MCVD process using primarily silicon tetrachloride $\left(\mathrm{SiCl}_{4}\right)$ and germanium tetrachloride $\left(\mathrm{GeCl}_{4}\right)$. Then, the silica glass tube with the deposited layers was dried and partially sintered. To incorporate Ge NPs in the porous core layers, the tube was soaked with a Ge-doping solution prepared by dissolving 0.1 mole of high purity Ge powders ( $45 \mu \mathrm{m}$ under, Kojundo, GEE05PB) in deionized water for two hours. Then, the Ge NP-doped glass tube was collapsed into a rod as a fiber preform. And the cladding part of the fiber preform was removed by etching with a hydrofluoric acid solution to obtain the core part of the Ge NP-doped glass with a diameter of $2 \mathrm{~mm}$ from the preform.

To fabricate a PCF preform with the Ge NP-doped glass core of $2 \mathrm{~mm}$ diameter, capillary tubes of silica glass with the same diameter of $2 \mathrm{~mm}(1.4 \times 2 \mathrm{~mm}$ diameters $)$ were prepared by drawing a silica glass tube $(19 \times 25 \mathrm{~mm}$ diameters $)$. The 60 capillary silica glass tubes were stacked 5 layers around the core rod in a hexagonal pattern, and it was jacketed with a large silica glass tube $(19 \times 25 \mathrm{~mm}$ diameters $)$ into a first PCF preform (PCF preform 1). Then, the first PCF preform was drawn into a cane with a diameter of $2 \mathrm{~mm}$, and the cane was jacketed again with a silica tube $(2 \times 6 \mathrm{~mm}$ diameters) into a second PCF preform (PCF preform 2). After all, this second PCF preform was drawn into a final PCF fiber of $125 \mu \mathrm{m}$ diameter with a Ge NP-doped glass core. Note that the pressure was applied to keep the air hole circular during the drawing process. The diameter of the core and the air hole, and the hole-to-hole distance (pitch, $\Lambda$ ) of the fabricated Ge NP-doped core PCF were $4.1 \mu \mathrm{m}$ and $2.9 \mu \mathrm{m}$, and $5.2 \mu \mathrm{m}$, respectively (Figure 1). For a comparison, a PCF with the pure silica glass core and a PCF with the germanosilicate glass core without Ge NPs were also fabricated. The diameter of the core and the air hole, and the hole-to-hole distance $(\Lambda)$ of the pure silica core PCF were $6.0 \mu \mathrm{m}$ and $1.9 \mu \mathrm{m}$, and $4.3 \mu \mathrm{m}$, respectively. And those of the PCF with the germanosilicate glass core without Ge NPs were $4.2 \mu \mathrm{m}$ and $3.3 \mu \mathrm{m}$ and $5.1 \mu \mathrm{m}$, respectively.

To verify the existence of Ge NPs in the core of the PCF, the fabricated fiber was examined by the Cs-corrected scanning TEM (JEOL, JEM-ARM200F). The optical absorption of the fiber was also measured by the cut-back method using the optical spectrum analyzer (OSA; Ando, AQ 6315B) and white light source (WLS; Ando, AQ 4303B). Note that the cut-back method is done by comparing the optical power transmitted through a long fiber to that transmitted through a short piece of the fiber after cutting the long fiber without variation of the input signal. Furthermore, to find optimum experimental condition for SCG, the chromatic dispersion and the nonresonant optical nonlinearity such as the nonresonant nonlinear refractive index coefficient, $n_{2}$, and the effective nonlinear parameter, $\gamma$, were obtained by using the optical dispersion analyzer (ODA; AGILENT, 86038B) and the continuous wave self-phase modulation (cw-SPM) method, respectively. A detail measurement method and the result of the PCF fiber were described in reference [2]. The estimated nonresonant nonlinear refractive index, $n_{2}$, and the effective nonlinear parameter, $\gamma$, of the Ge NPdoped core PCF were found to be $4.74 \times 10^{-20} \mathrm{~m}^{2} / \mathrm{W}$ and $14.41 \mathrm{~W}^{-1} \mathrm{~km}^{-1}$, respectively [27]. And the $n_{2}$ and the $\gamma$ of the pure silica core PCF and the germanosilicate glass core without Ge NPs were $2.56 \times 10^{-20} \mathrm{~m}^{2} / \mathrm{W}$ and $3.67 \mathrm{~W}^{-1} \mathrm{~km}^{-}$ ${ }^{1}$, and $2.62 \times 10^{-20} \mathrm{~m}^{2} / \mathrm{W}$ and $7.82 \mathrm{~W}^{-1} \mathrm{~km}^{-1}$, respectively. The $n_{2}$ and $\gamma$ of the Ge NP-doped core PCF were larger than those of the PCFs with the pure silica core and the germanosilicate glass core without Ge NPs. The specifications of the fabricated PCFs are summarized in Table 1.

SCG was obtained by pumping the $5 \mathrm{~m}$ long fiber with the Ti:sapphire femtosecond laser (Spectra-Physics, Spitfire Ace) at $800 \mathrm{~nm}$ with the power of $160 \mathrm{~mW}$ and the pulse width of $35 \mathrm{fs}$ at $1 \mathrm{kHz}$ repetition rate. The output spectrum of the SCG was measured by using the optical spectrum analyzer (OSA: ANDO, AQ-6315B). Note that the pumping laser was directly launched onto the fiber core using a focusing lens.

\section{Results and Discussion}

Figure 2 shows the TEM image and the size distribution of $\mathrm{Ge}$ NPs embedded in the germanosilicate glass core of the PCF. It clearly shows the existence of the roughly spherical crystalline Ge NPs even after the high temperature drawing process of over $1950^{\circ} \mathrm{C}$. The average diameter of Ge NPs was measured to be about $4.2 \mathrm{~nm}$ with the range of $3.4 \mathrm{~nm} \sim 5.4 \mathrm{~nm}$. Despite the multiple high temperature drawing processes (the $2 \mathrm{~mm}$ rod drawing, the cane drawing, and the final fiber drawing), Ge NPs were well retained in the core of the PCF. The existence of Ge NPs in the core of the PCF was also verified by absorption spectrum analysis. As shown in Figure 3(a), two absorption peaks appeared at $480 \mathrm{~nm}$ and $515 \mathrm{~nm}$, and an absorption band appeared centering at $650 \mathrm{~nm}$, and these are attributed to Ge NPs in the core of the PCF [23, 27]. An absorption peak at $1380 \mathrm{~nm}$ is due to the presence of $\mathrm{OH}$ ion impurities.

To estimate an optimum wavelength for the SCG of the PCF, the chromatic dispersion of the Ge NP-doped core PCF was measured and shown in Figure 3(b). The measured zero-dispersion wavelength was $1246.3 \mathrm{~nm}$, a little shorter than the simulated zero-dispersion wavelength of $1361.0 \mathrm{~nm}$ by using the finite element method- (FEM-) based COMSOL program [27]. Even though pumping for an efficient SCG must be carried out near the zero-dispersion wavelength, the actual pumping was done at $800 \mathrm{~nm}$ (normal dispersion of $D \approx-1238.9 \mathrm{ps} / \mathrm{nm} / \mathrm{km}$ ) by using the available commercial high-power femtosecond laser [16]. The laser 


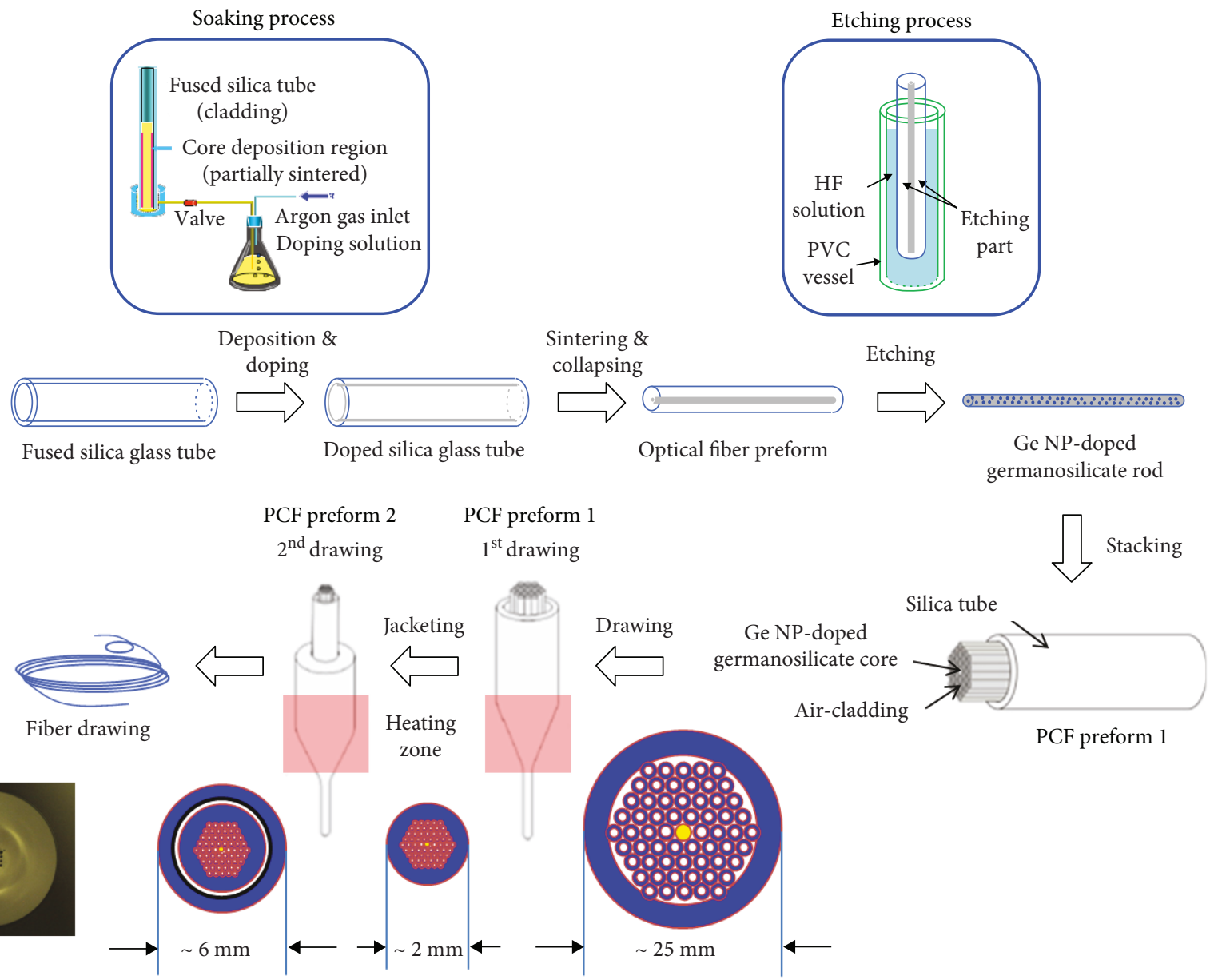

FIGURE 1: Fabrication process and fabricated cross-section image of the Ge NP-doped core PCF.

TABLE 1: Specifications of the fabricated PCFs.

\begin{tabular}{|c|c|c|c|c|c|c|}
\hline Fiber & $\begin{array}{l}\text { Solid core } \\
\text { diameter }\end{array}$ & $\begin{array}{l}\text { Air hole } \\
\text { diameter }\end{array}$ & $\begin{array}{l}\text { Hole-to-hole } \\
\text { distance (pitch) }\end{array}$ & $\begin{array}{l}\text { Chromatic dispersion } \\
\text { coefficient @ } 800 \mathrm{~nm}\end{array}$ & $\begin{array}{l}\text { Nonlinear } \\
\text { refractive index }\end{array}$ & $\begin{array}{c}\text { Effective } \\
\text { nonlinearity }\end{array}$ \\
\hline Symbol & - & - & $\Lambda$ & $D$ & $n_{2}$ & $\gamma$ \\
\hline Unit & $(\mu \mathrm{m})$ & $(\mu \mathrm{m})$ & $(\mu \mathrm{m})$ & $(\mathrm{ps} / \mathrm{nm} / \mathrm{km})$ & $\left(\mathrm{m}^{2} / \mathrm{W}\right)$ & $\left(\mathrm{W}^{-1} \mathrm{~km}^{-1}\right)$ \\
\hline Pure silica core PCF & 6.0 & 1.9 & 4.3 & -932.5 & $2.56 \times 10^{-20}$ & 3.67 \\
\hline $\begin{array}{l}\text { Germanosilicate glass core PCF } \\
\text { (without Ge NPs) }\end{array}$ & 4.2 & 3.3 & 5.1 & -1073.5 & $2.62 \times 10^{-20}$ & 7.82 \\
\hline $\begin{array}{l}\text { Ge NP-doped germanosilicate } \\
\text { glass core PCF }\end{array}$ & 4.1 & 2.9 & 5.2 & -1238.9 & $4.74 \times 10^{-20}$ & 14.41 \\
\hline
\end{tabular}

was launched onto the core of the $5 \mathrm{~m}$ long PCFs at the pump power of $160 \mathrm{~mW}$ with $35 \mathrm{fs}$ pulse width at $1 \mathrm{kHz}$ repetition.

Figure 4 compares the output powers of the PCFs upon pumping with the Ti:sapphire femtosecond laser at $800 \mathrm{~nm}$. All the PCFs showed the SCG from $600 \mathrm{~nm}$ to $1100 \mathrm{~nm}$ after pumping with the laser. The SCG bandwidth of the Ge NPdoped core PCF was wider than that of the pure silica glass core PCF and the germanosilicate glass core PCF. The bandwidth at the output power of $-60 \mathrm{dBm}$ of the SC spectrum (full width at half maximum, FWHM) of the pure silica core PCF and the germanosilicate glass core PCF at the same output power were $393 \mathrm{~nm}(650 \mathrm{~nm} \sim 1043 \mathrm{~nm})$ and $414 \mathrm{~nm}$
$(640 \mathrm{~nm} \sim 1054 \mathrm{~nm})$, respectively. But that of the Ge NPdoped core PCF was $490 \mathrm{~nm}(598 \mathrm{~nm} \sim 1088 \mathrm{~nm})$. The average power from the SC spectrum of the Ge NP-doped core PCF was $49.6 \mathrm{~mW}$ with a power conversion efficiency of $31 \%$. It is known that when the PCFs are pumped at wavelengths of normal dispersion region (shorter than zero-dispersion wavelength), which is our case, spectral broadenings are narrow and smooth, and it is due to the self-phase modulation only. On the other hand, when the PCFs are pumped at wavelengths of anomalous dispersion region (longer than zero-dispersion wavelength), dominant nonlinear processes became modulation instability and 


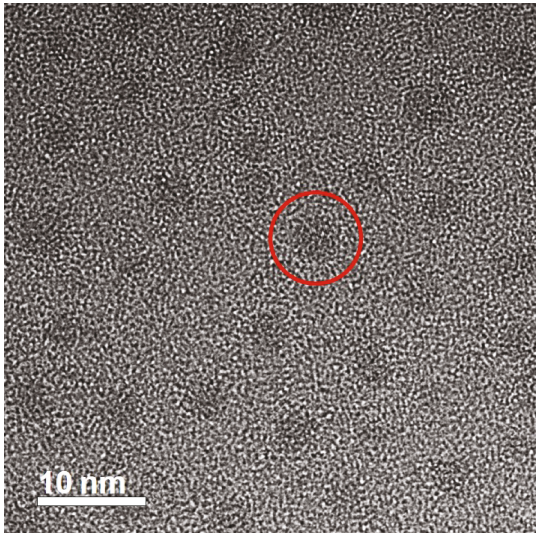

(a)

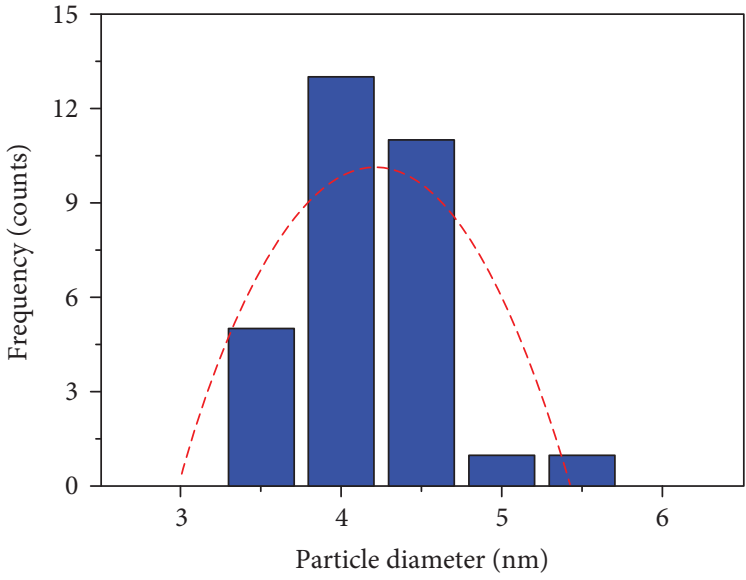

(b)

FIgURe 2: (a) TEM image and (b) size distribution of Ge NPs in the core of the Ge NPs-doped core PCF.

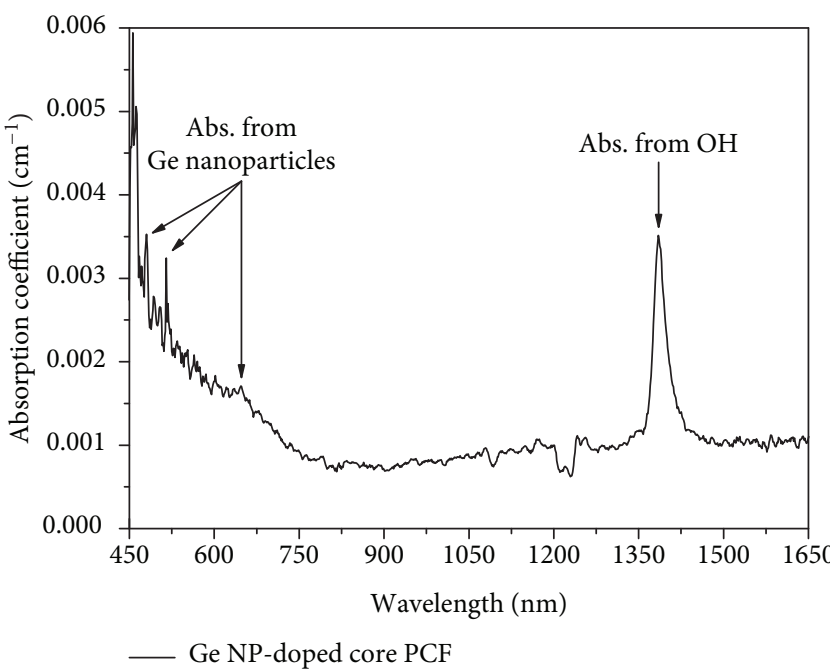

(a)

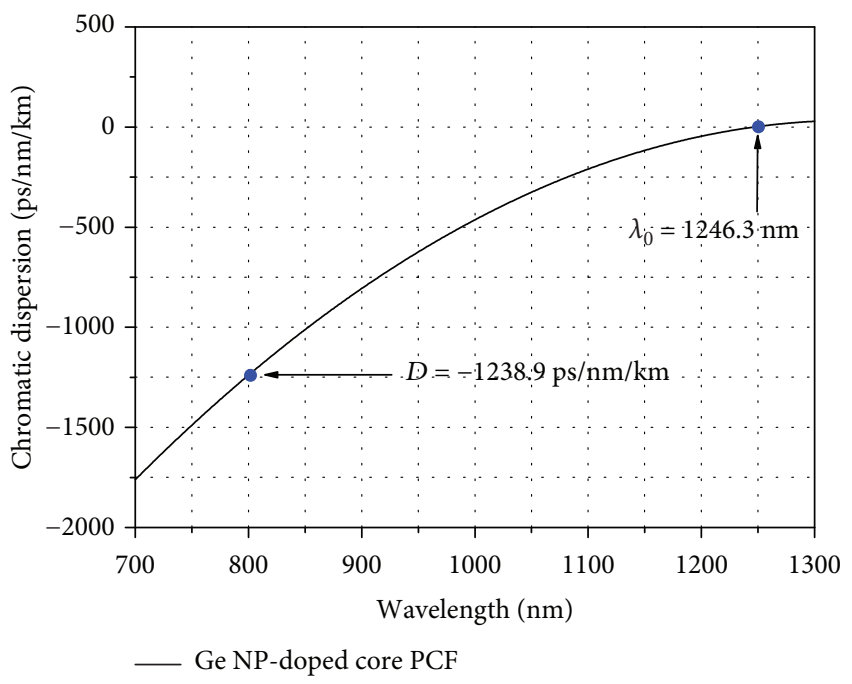

(b)

FIgURE 3: (a) Optical absorption spectrum and (b) chromatic dispersion of the Ge NP-doped core PCF.

solitonal dynamics, and thus, the output spectral bandwidth are wide and rough [16].

Particularly, the expansion of the SCG bandwidth of the Ge NP-doped core PCF compared to the others (from 393 and $414 \mathrm{~nm}$ to $490 \mathrm{~nm}$ ) demonstrates the role of Ge NPs to the large optical nonlinearity. The nonresonant optical nonlinearity of the Ge NP-doped core PCF may be caused by the hyperpolarizabilities of NBOs formed by the incorporated Ge NPs, and this NBOs have higher ionicity and are easily distorted by the applied optical field $[23,27]$. In the viewpoint of the PCF structure, the germanosilicate core PCF has a broader SC spectrum than the pure silica core PCF because the small core diameter causes an increase in optical nonlinearity [29]. Note that as shown in Table 1, the $\gamma$ of the germanosilicate glass core PCF was larger than that of the pure silica glass core PCF because of the core size difference while the $n_{2}$ of both PCFs was almost the same.

\section{Conclusions}

The effect of Ge NP incorporation in the germanosilicate glass core of the PCF on SCG was investigated. Three types of PCFs, the Ge NP-doped germanosilicate glass core PCF, the germanosilicate glass core PCF, and the pure silica glass core PCF, were fabricated by using the MCVD process and the stack-and-draw technique. The diameters of the core and the air hole, and the hole-to-hole distance $(\Lambda)$ of the fabricated Ge NP-doped core PCF were $6.0 \mu \mathrm{m}$ and $1.9 \mu \mathrm{m}$, and $4.3 \mu \mathrm{m}$, respectively. The average diameter of Ge NPs embedded in the core of the PCF was $4.2 \mathrm{~nm}(3.4 \mathrm{~nm} \sim 5.4 \mathrm{~nm})$. The absorption peaks appearing at $480 \mathrm{~nm}$ and $515 \mathrm{~nm}$ and the band centering at $650 \mathrm{~nm}$ were attributed to the Ge NPs in the core of the PCF. The measured zero-dispersion wavelength and the normal dispersion at $800 \mathrm{~nm}$ were at $1246.3 \mathrm{~nm}$ and $-1238.9 \mathrm{ps} / \mathrm{nm} / \mathrm{km}$, respectively. And the nonresonant nonlinear refractive index $\left(n_{2}\right)$ and the effective 


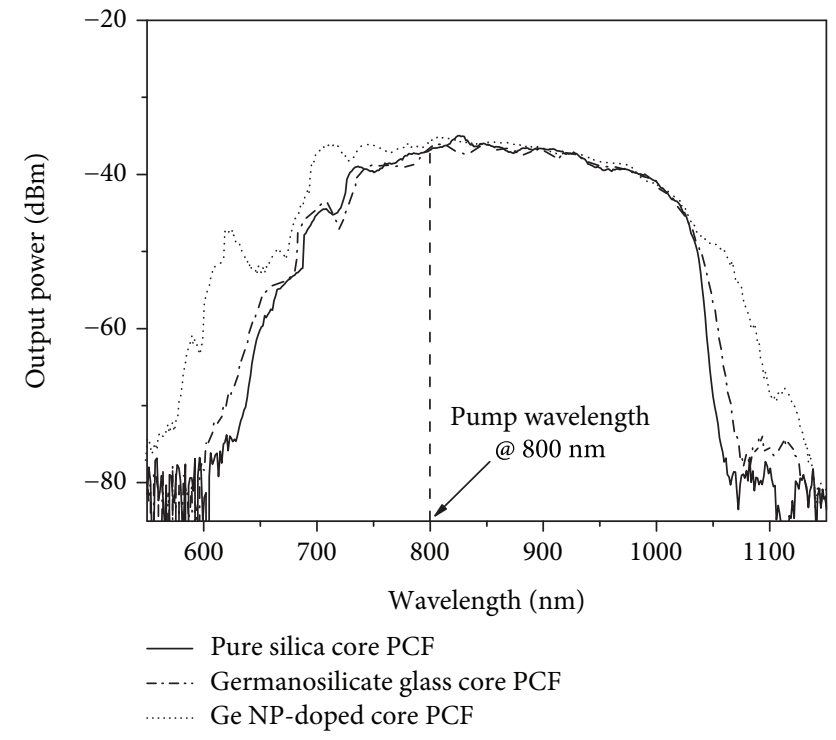

FIGURE 4: Supercontinuum generation of the PCFs upon pumping with the femtosecond laser at $800 \mathrm{~nm}$.

nonlinear parameter $(\gamma)$ of the Ge NP-doped core PCF measured by the cw-SPM method were $4.74 \times 10^{-20}$ $\mathrm{m}^{2} / \mathrm{W}$ and $14.41 \mathrm{~W}^{-1} \mathrm{~km}^{-1}$, respectively. SCG was obtained by pumping into the fiber core at $800 \mathrm{~nm}$ with the Ti:sapphire femtosecond laser of $160 \mathrm{~mW}$ with $35 \mathrm{fs}$ pulse at $1 \mathrm{kHz}$ repetition rate. The SCG bandwidth at the output power of $-60 \mathrm{dBm}$ of the Ge NP-doped core PCF was $490 \mathrm{~nm}(598 \mathrm{~nm} 1088 \mathrm{~nm})$ with a conversion efficiency of $31 \%$, resulting from the enhanced optical nonlinearity from Ge NPs as well as the PCF structure of the fiber.

\section{Data Availability}

The data used to support the findings of this study are available from the corresponding author upon request.

\section{Conflicts of Interest}

The authors declare that they do not have conflicts of interest.

\section{Acknowledgments}

This work was partially supported by the KEPCO Research Institute of South Korea under the grant number KEPRI16-23.

\section{References}

[1] J. M. Dudley, G. Genty, and S. Coen, "Supercontinuum generation in photonic crystal fiber," Reviews of Modern Physics, vol. 78, no. 4, pp. 1135-1184, 2006.

[2] G. Humbert, W. Wadsworth, S. Leon-Saval et al., "Supercontinuum generation system for optical coherence tomography based on tapered photonic crystal fibre," Optics Express, vol. 14, no. 4, pp. 1596-1603, 2006.
[3] A. Bassi, J. Swartling, C. D’Andrea, A. Pifferi, A. Torricelli, and R. Cubeddu, "Time-resolved spectrophotometer for turbid media based on supercontinuum generation in a photonic crystal fiber," Optics Letters, vol. 29, no. 20, pp. 2405-2407, 2004.

[4] T. Morioka, K. Mori, and M. Saruwatari, "More than 100wavelength-channel picosecond optical pulse generation from single laser source using supercontinuum in optical fibres," Electronics Letters, vol. 29, no. 10, pp. 862-864, 1993.

[5] J. M. Dudley and J. R. Taylor, "Ten years of nonlinear optics in photonic crystal fibre," Nature Photonics, vol. 3, no. 2, pp. 8590, 2009.

[6] T. A. Birks, W. J. Wadsworth, and P. S. J. Russell, "Supercontinuum generation in tapered fibers," Optics Letters, vol. 25, no. 19, pp. 1415-1417, 2000.

[7] R. Halir, Y. Okawachi, J. S. Levy, M. A. Foster, M. Lipson, and A. L. Gaeta, "Ultrabroadband supercontinuum generation in a CMOS-compatible platform," Optics Letters, vol. 37, no. 10, pp. 1685-1687, 2012.

[8] A. Martinez and S. Yamashita, "Multi-gigahertz repetition rate passively modelocked fiber lasers using carbon nanotubes," Optics Express, vol. 19, no. 7, pp. 6155-6163, 2011.

[9] T. Udem, R. Holzwarth, and T. W. Hänsch, "Optical frequency metrology," Nature, vol. 416, no. 6877, pp. 233-237, 2002.

[10] T. Morioka, H. Takara, S. Kawanishi et al., "1 Tbit/s (100 Gbit/s $\times 10$ channel) OTDM/WDM transmission using a single supercontinuum WDM source," Electronics Letters, vol. 32, no. 10 , p. 906, 1996.

[11] T. Kuri, T. Nakasyotani, H. Toda, and K.-I. Kitayama, "Characterizations of supercontinuum light source for WDM millimeter-wave-band radio-on-fiber systems," IEEE Photonics Technology Letters, vol. 17, no. 6, pp. 1274-1276, 2005.

[12] A. L. Gaeta, "Catastrophic collapse of ultrashort pulses," Physical Review Letters, vol. 84, no. 16, pp. 3582-3585, 2000.

[13] P. B. Corkum, P. P. Ho, R. R. Alfano, and J. T. Manassah, "Generation of infrared supercontinuum covering 3-14 $\mu \mathrm{m}$ in dielectrics and semiconductors," Optics Letters, vol. 10, no. 12, pp. 624-626, 1985.

[14] S. Jeong, S. Ju, Y. Kim et al., "Effective supercontinuum generation by using highly nonlinear dispersion-shifted fiber incorporated with Si nanocrystals," Journal of Nanoscience and Nanotechnology, vol. 12, no. 1, pp. 458-462, 2012.

[15] P. S. J. Russell, "Photonic-crystal fibers," Journal of Lightwave Technology, vol. 24, no. 12, pp. 4729-4749, 2006.

[16] W. Q. Zhang, H. Ebendorff-Heidepriem, T. M. Monro, and S. V. Afshar, "Fabrication and supercontinuum generation in dispersion flattened bismuth microstructured optical fiber," Optics Express, vol. 19, no. 22, pp. 21135-21144, 2011.

[17] J. H. V. Price, T. M. Monro, H. Ebendorff-Heidepriem et al., "Mid-IR supercontinuum generation from nonsilica microstructured optical fibers," IEEE Journal of Selected Topics in Quantum Electronics, vol. 13, no. 3, pp. 738-749, 2007.

[18] J. Y. Y. Leong, P. Petropoulos, J. H. V. Price et al., "Highnonlinearity dispersion-shifted lead-silicate holey fibers for efficient $1-\mu \mathrm{m}$ pumped supercontinuum generation," Journal of Lightwave Technology, vol. 24, no. 1, pp. 183-190, 2006.

[19] H. J. Cho, A. Lin, S. Moon, W.-T. Han, and B. H. Kim, "Nonresonant optical nonlinearity of germano-silicate optical fiber incorporated with Si nanocrystals," Journal of the Korean Physical Society, vol. 53, no. 3, pp. 1565-1569, 2008. 
[20] S. Das, R. Aluguri, S. Manna et al., "Optical and electrical properties of undoped and doped Ge nanocrystals," Nanoscale Research Letters, vol. 7, no. 1, p. 143, 2012.

[21] L. E. Ramos, H.-C. Weissker, J. Furthmüller, and F. Bechstedt, "Optical properties of Si and Ge nanocrystals: parameter-free calculations," Physica Status Solidi (b), vol. 242, no. 15, pp. 3053-3063, 2005.

[22] M. Onishi, T. Okuno, T. Kashiwada, S. Ishikawa, N. Akasaka, and M. Nishimura, "Highly nonlinear dispersion-shifted fibers and their application to broadband wavelength converter," Optical Fiber Technology, vol. 4, no. 2, pp. 204-214, 1998.

[23] S. Jeong, S. Ju, Y. Kim, H. Jeong, S. Boo, and W. T. Han, "Development of germano-silicate optical fiber incorporated with germanium nanoparticles and its optical characteristics," Journal of Nanoscience and Nanotechnology, vol. 16, no. 6, pp. 6378-6382, 2016.

[24] M. Avella, A. C. Prieto, J. Jiménez, A. Rodríguez, J. Sangrador, and T. Rodríguez, "Violet luminescence in Ge nanocrystals/Ge oxide structures formed by dry oxidation of polycrystalline SiGe," Solid State Communications, vol. 136, no. 4, pp. 224227, 2005.

[25] C. Bostedt, T. van Buuren, T. M. Willey et al., "Strong quantum-confinement effects in the conduction band of germanium nanocrystals," Applied Physics Letters, vol. 84, no. 20, pp. 4056-4058, 2004.

[26] E. G. Barbagiovanni, D. J. Lockwood, P. J. Simpson, and L. V. Goncharova, "Quantum confinement in Si and Ge nanostructures: theory and experiment," Applied Physics Reviews, vol. 1, no. 1, article 011302, 2014.

[27] S. Jeong, S. Ju, Y. Lee et al., "A novel photonic crystal fiber with a germanium nanoparticles-doped germano-silicate core and its nonlinear optical characteristics," in , Article ID 103234L25th International Conference on Optical Fiber Sensors, vol. 10323, Jeju, South Korea, April 2017.

[28] S. R. Nagel, J. B. MacChesney, and K. L. Walker, "An overview of the modified chemical vapor deposition (MCVD) process and performance," IEEE Transactions on Microwave Theory and Techniques, vol. 30, no. 4, pp. 305-322, 1982.

[29] G. Brambilla, F. Koizumi, V. Finazzi, and D. J. Richardson, "Supercontinuum generation in tapered bismuth silicate fibres," Electronics Letters, vol. 41, no. 14, pp. 795-797, 2005. 


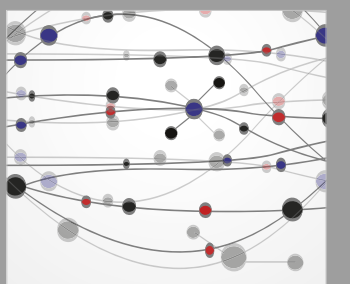

The Scientific World Journal
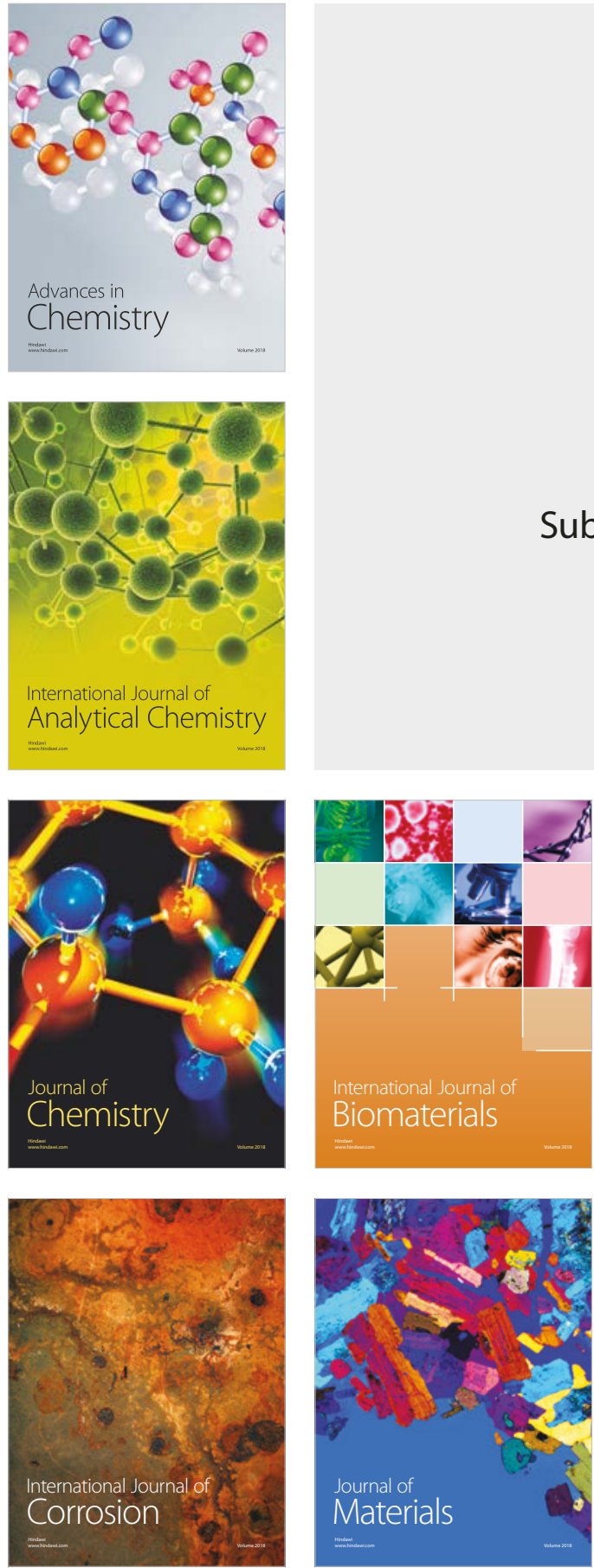

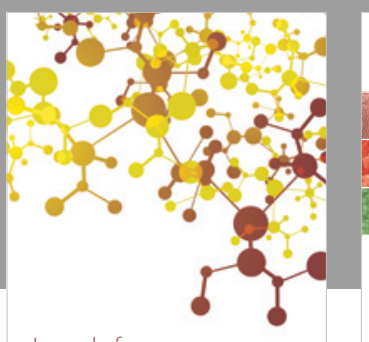

Journal of

Applied Chemistry
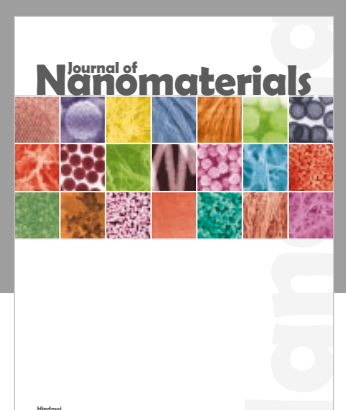

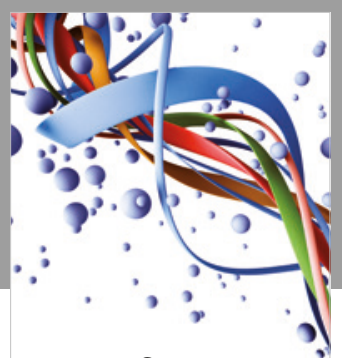

Scientifica

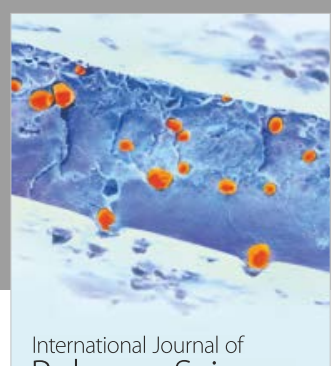

Polymer Science

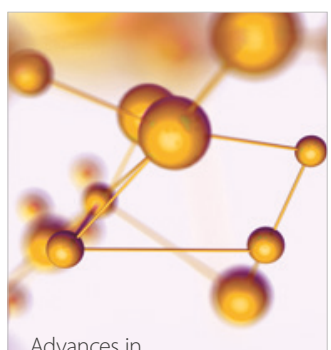

Physical Chemistry
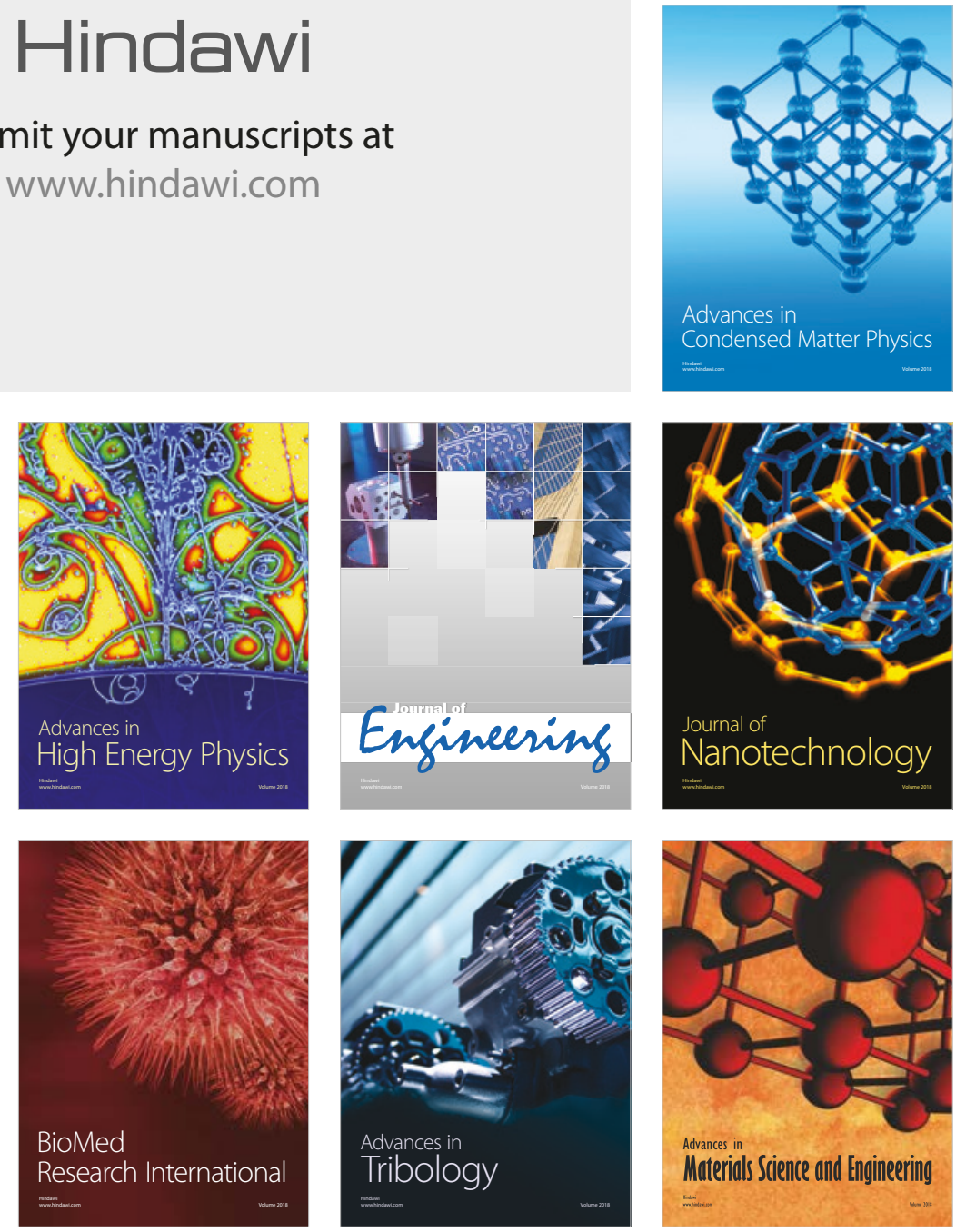